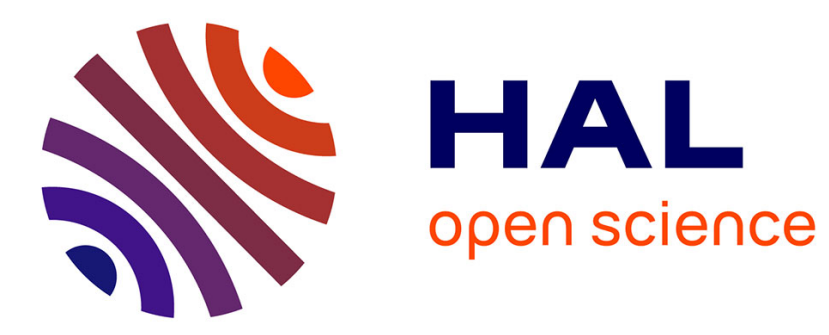

\title{
THERMAL DIFFUSION IN LENNARD-JONES FLUIDS IN THE FRAME OF THE LAW OF THE CORRESPONDING STATES
}

Guillaume Galliero

\section{- To cite this version:}

Guillaume Galliero. THERMAL DIFFUSION IN LENNARD-JONES FLUIDS IN THE FRAME OF THE LAW OF THE CORRESPONDING STATES. Fluid Phase Equilibria, 2004, 224 (1), pp.13-22. 10.1016/j.fluid.2004.06.040 . hal-00322215

\section{HAL Id: hal-00322215 https://hal.science/hal-00322215}

Submitted on 17 Sep 2008

HAL is a multi-disciplinary open access archive for the deposit and dissemination of scientific research documents, whether they are published or not. The documents may come from teaching and research institutions in France or abroad, or from public or private research centers.
L'archive ouverte pluridisciplinaire HAL, est destinée au dépôt et à la diffusion de documents scientifiques de niveau recherche, publiés ou non, émanant des établissements d'enseignement et de recherche français ou étrangers, des laboratoires publics ou privés. 


\title{
THERMAL DIFFUSION IN LENNARD-JONES FLUIDS IN THE FRAME OF THE LAW OF THE CORRESPONDING STATES.
}

\author{
Guillaume Galliéro ${ }^{1,2}$ \\ ${ }^{1}$ Laboratoire TREFLE, site ENSCPB, UMR 8508 with CNRS, Université de Bordeaux \\ I, 16 Avenue Pey-Berland, 33607 Pessac Cedex, FRANCE. \\ ${ }^{2}$ Laboratoire des Fluides Complexes, Université de Pau et des Pays de l'Adour, UMR \\ 5150 with CNRS, Centre Universitaire de Recherche Scientifique, B.P. 1155, 64013 \\ Pau Cedex, FRANCE. \\ Tel : +33-5-40-00-28-31; fax : +33-5-40-00-66-68; \\ Email: galliero@enscpb.fr
}

Keywords: nonequilibrium molecular dynamics, molecular simulation, thermal diffusion, mixing rules, corresponding states, Lennard-Jones mixture.

\begin{abstract}
This work is related to the definition of a reduced thermal diffusion coefficient thanks to numerical microscale molecular dynamics simulations. This cross transport process, also called Soret effect, couples mass flux and thermal gradient and is still largely misunderstood. For this study, we have applied a boundary driven non equilibrium molecular dynamics algorithm on Lennard-Jones spheres mixtures. Simulations have been performed at a constant reduced supercritical state, using a van der Waals one fluid approximation in order to fulfil the law of the corresponding states. In binary mixtures, we have studied the molecular parameters and the molar fraction influences on thermal diffusion separately and then combined. It is shown that, on pressure and on thermal conductivity, the corresponding states law is fulfilled for a wide range of molecular
\end{abstract}


parameters ratios. In this frame, we have then constructed simple correlations which relate thermal diffusion factor to the mixture parameters. Combining the relations obtained, a reduced thermal diffusion factor taking into account all the various contributions has been defined. Finally, it is shown that this relation enables us to estimate thermal diffusion in various binary and ternary mixtures of Lennard-Jones spheres representing alkanes with a maximum deviation of $15 \%$.

\section{Introduction}

A good knowledge of the thermal diffusion coefficients, also called Soret effect in condensed phase, in hydrocarbon mixtures is required in the oil and gas industry to ensure reliable formation fluid evaluation [1]. But, in that case, the huge number of components implies the use of simple and efficient models. This cross transport process couples mass flux and thermal gradient and is usually weak compared to classical mass diffusion. Even if this phenomenon has been studied for a long time, its complete physical explanation is still missing [2]. However, some recent improvements in the modelling [3-4] and in the measurements and comprehension have been achieved [5].

Nowadays, molecular simulation appears to be an alternative to the experiment for the evaluation of transport and thermodynamics properties of pure fluids and simple mixtures. This is particularly true when dealing with experimentally inaccessible thermodynamics conditions or with weak phenomena such as thermal diffusion. In particular, molecular dynamics reveals itself to be a useful tool to improve our understanding of the physical processes that govern this transport phenomenon [2, 6-9] which is a compulsory step to build reliable correlations based on the law of the corresponding states [10] for simple mixtures. 
The use of the corresponding states law, applied thanks to a one fluid approximation when dealing with mixtures, is a crucial point because it enables us, for a given transport property, to uncouple the thermodynamics state contribution from the molecular parameters one[11]. It is then possible, for a given reduced thermodynamics state, to study specifically the mixture parameters influence on a transport property. The Lennard-Jones (LJ) sphere model which implies only three parameters to define a molecule, its mass, its atomic diameter and its potential strength, is particularly adapted to such a study. Concerning the one fluid approximation, we have used the van der Walls one (vdW1) which is simple and efficient [12].

So, for a given thermodynamics state in reduced variables, we have performed simulations on Lennard-Jones equimolar and nonequimolar mixtures to first assess the validity of the one fluid approximation chosen for the thermal conductivity and the pressure of the studied mixture. Then, following previous works $[9,13]$, we have defined the relevant variables that correlate the thermal diffusion factor to the various molecular parameters in equimolar mixture. It is shown that these various contributions can be combined into a single relation taking into account the couplings. From this point, a reduced thermal diffusion factor has been defined using the previous results. This correlation has been applied on several binary mixtures, sometimes having a large discrepancy between molecular parameters, and the maximum deviation between computation and prediction has been found to reach $15 \%$.

The influence of the molar fraction has been analyzed and correlated as well. In alkane mixtures it is shown that only the equimolar thermal diffusion value is needed to estimate correctly this property for the whole set of molar fractions. This correlation is able to provide a result which does not deviate from the computed value from more than $15 \%$. 
Combining these results to define a comprehensive expression of a reduced thermal diffusion factors, it is then possible to achieve a consistent estimation of the thermal diffusion for nonequimolar alkane binary and ternary mixtures starting only from the mixture parameters.

\section{Models and methods used}

Thermodynamics of Irreversible Process (TIP) provides a formalism to describe the couplings between the different kinds of transport in multicomponents systems [14]. Such a description linearly links the fluxes with the thermodynamics forces. According to the principles of TIP, when a non reacting binary mixture in mechanical equilibrium is subjected to a temperature gradient, the phenomenological mass flux $\mathbf{J}_{m}$ of one of the species $i$ is,

$$
\mathbf{J}_{m}=-\rho D_{12} \nabla w_{i}-\rho w_{i}\left(1-w_{i}\right) D_{T} \nabla T
$$

where $D_{12}$ and $D_{T}$ are respectively the mutual and the thermal diffusion coefficients of the mixture, $\rho$ the average density, $w_{i}$ the mass fraction of the studied component and $T$ the temperature. If a temperature gradient is applied to a homogeneous mixture in mechanical equilibrium without gravity, the system is driven, after a transient state, toward a nonequilibrium steady state where the mass flux vanishes $\left(\mathbf{J}_{m}=0\right)$. In this steady state, the two contributions to the mass flux in equation (1), due to mutual diffusion and to thermal diffusion, balance each other. Rewriting equation (1) at the steady state multiplied by the average temperature, we obtain then:

$$
T \frac{D_{T}}{D_{12}}=-\frac{T}{w_{i}\left(1-w_{i}\right)} \frac{\nabla w_{i}}{\nabla T}=\alpha_{T i}
$$

This relation gives us an expression for the thermal diffusion factor $\alpha_{T i}$ that characterizes the amplitude of the phenomena. In binary mixture this relation is strictly equivalent to the one defined using molar fraction, $x_{i}$, instead of mass fraction. In binary 
mixtures, $\alpha_{T 1}$ will be noted for simplicity $\alpha_{T}$. For ternary mixtures we have adopted the formalism of [3] which defined the thermal diffusion factor of a component $i$ as:

$$
\alpha_{T i}=-\frac{T}{x_{i}\left(1-x_{i}\right)} \frac{\nabla x_{i}}{\nabla T}
$$

The fluid components are described by smooth spheres, without any internal degree of freedom, interacting through a classical truncated LJ 12-6 potential [15],

$$
U_{L J}=4 \varepsilon_{i j}\left(\left(\frac{\sigma_{i j}}{r_{i j}}\right)^{12}-\left(\frac{\sigma_{i j}}{r_{i j}}\right)^{6}\right)
$$

where the subscripts $i$ and $j$ characterize the species. In this picture only three parameters are needed to describe a molecule, its molar mass $m$, its atomic diameter $\sigma$ and its potential depth $\varepsilon$.

Concerning the interactions between unlike particles, the classical Lorentz-Berthelot combining rules have been applied.

In order to study the thermal transport properties, we have used a Boundary Driven Non Equilibrium Molecular Dynamics (BD-NEMD) code of our own. This method consists in applying a periodical thermal gradient to the simulation box by exchanging kinetic energy between edge layers. The algorithm chosen to generate the thermal gradient in the simulation box is the one proposed by Hafskjold [2]. This efficient algorithm [16], allows us to obtain directly the thermal conductivity and the thermal diffusion factor in one simulation without any approximation on the mixture nature.

We have used a time step of 5 fs and a cut off radius equal to $2.5 \sigma$. The simulations have been applied on to 1500 centres of force. A reduced energy flux of $8.3510^{-2}$ has been used in the nonequilibrium simulations. Moreover, results provided correspond to simulations during at least $10^{6}$ time steps to ensure a reliable estimation of thermal diffusion [16]. 
Our code has shown to yield consistent values for noble gases and for simple alkane mixtures compared to experimental ones found in literature $[9,17]$.

In order to perform simulations in the law of the corresponding states' scheme, it is necessary to work with reduced thermodynamics variables. When using LJ spheres, these variables are defined as:

$$
T^{*}=\frac{k_{b} T}{\varepsilon_{x}}, \rho^{*}=\frac{N \sigma_{x}^{3}}{V} \text { and } P^{*}=\frac{P \sigma_{x}^{3}}{\varepsilon_{x}}
$$

where $k_{b}$ is the Boltzmann constant, $N$ the number of particles, $V$ the volume and $P$ the pressure.

The LJ parameters noted with a subscript $x$ are characteristic values of the molecular parameters of the studied fluid. In mixtures they must be defined thanks to a one fluid approximation. To do so, we have selected the van der Waals one fluid approximation which is efficient and simple [12]. In a $K$-components mixture the LJ molecular parameters in the vdW1 approximation are defined as:

$$
\begin{gathered}
\sigma_{x}^{3}=\sum_{i=1}^{K} \sum_{j=1}^{K} x_{i} x_{j} \sigma_{i j}^{3} \\
\varepsilon_{x} \sigma_{x}^{3}=\sum_{i=1}^{K} \sum_{j=1}^{K} x_{i} x_{j} \varepsilon_{i j} \sigma_{i j}^{3}
\end{gathered}
$$

When the law of the corresponding states is fulfilled for the model fluid, it is possible to formulate that a transport property is a function of two thermodynamics variables, in reduced units, and of the three LJ molecular parameters. Thus the thermodynamics contribution can be split from the molecular one and a transport property, $\tau$, can be written as:

$$
\tau=\tau^{*}\left(T^{*}, \rho^{*}\right) \times z_{\tau}\left(\sigma_{x}, \varepsilon_{x}, m_{x}\right)
$$

where 


$$
m_{x}=\sum_{i=1}^{K} x_{i} m_{i}
$$

and function $z$ is a characteristic function of the transport property [11], $m_{i}$ being the molecular mass of component $i$. Nevertheless, when applied on real fluids, this scheme is appropriate for simple species only (e.g.: noble gases) and needs the introduction of, at least, another variable to deal with more complex components [10].

For the thermal conductivity the function $z$ is [11]:

$$
z_{\kappa}\left(\sigma_{x}, \varepsilon_{x}, M_{x}\right)=k_{b} \sigma_{x}^{-2} \varepsilon_{x}^{1 / 2} m_{x}^{-1 / 2}
$$

To our knowledge, such a function does not exist for thermal diffusion apart from dilute gases [18], even if some tries have been attempted [6]. Elsewhere, if such a relation in dense phases exists, this function should not be expressed in terms of the characteristic molecular parameters defined by equation (7-11) contrary to the others transport properties, because the amplitude of thermal diffusion depends on the discrepancy between the molecular parameters of the involved components and not on the characteristic parameters defined for example by (6), (7) and (9). This work provides a first step toward the establishment of such a function $z$ for thermal diffusion.

\section{Results and discussion}

A disadvantage about using a BD-NEMD algorithm is that a huge thermal gradient (around $10 \mathrm{~K} \cdot \mathrm{nm}^{-1}$ ) is needed to compute the thermal properties correctly [2]. Thereby a great care must be taken when studying mixtures close to phase transitions [16]. Thus, simulations have been performed in supercritical conditions at $T^{*}=2.273$ and $\rho^{*}=0.4227$, this thermodynamics state being far enough from the critical point located for a LJ fluid model at $T_{c}{ }^{*}=1.31$ and $\rho_{c}{ }^{*}=0.31$ [19]. This state has been chosen because both thermodynamics and kinetics effects are not negligible. 
For this reduced thermodynamics state, we have performed simulations on various binary and ternary mixtures in a lot of different configurations in order to extract the pertinent variables that influence thermal diffusion in LJ spheres mixtures. Moreover, we have evaluated the deviations from the law of the corresponding states induced by the van der Waals one fluid approximation for these mixtures.

For the methane we have taken the molecular parameters values given in [20], namely $m=16$ g.mol ${ }^{-1}, \sigma=0.3723 \mathrm{~nm}$ and $\varepsilon=1.2097 \mathrm{~kJ} \cdot \mathrm{mol}^{-1}$. For this pure component, the reduced thermodynamics state chosen corresponds to $T=330.8 \mathrm{~K}, \rho=217.6 \mathrm{~kg} \cdot \mathrm{m}^{-3}$ and the computed pressure gives $P=39.6 \pm 1 \% \mathrm{MPa}$, which, in reduced units, is $P^{*}=1.018 \pm 1 \%$. Concerning the thermal conductivity we have obtained $\kappa^{*}=2.173 \pm 2 \%$. These results are consistent with those coming from [21].

The statistical error bars have been omitted on figures, but they are about $1 \%$ for the pressures, $2 \%$ for the thermal conductivities and between $5 \%$ and $10 \%$ for the thermal diffusion factors.

\subsection{Separate influences in equimolar mixtures}

To analyse the influence of the three LJ molecular parameters, we have performed simulations on equimolar mixtures where one parameter of component 1 is changed at a time, while the others are taken equal to those of component 2 which have been taken equal to those of methane. Using this scheme enables us to study the uncoupled influences of the three molecular parameters on thermal diffusion.

We have achieved this systematic study by evaluating the pressure, the thermal conductivity and the thermal diffusion factor for a wide range of molecular parameters ratios between the two synthetic species. The molecular parameters ratio goes from 0.5 to 500 for the mass, 0.75 to 5 for the potential strength and from 0.75 to 4 for the atomic 
diameter. The results of the computed reduced pressure and reduced thermal conductivities versus the molecular parameters ratios are shown on figures (1) to (3).

Generally, the deviation from the law of the corresponding states, using the van der Waals one fluid approximation, increases with the molecular parameters ratios.

On one hand, these figures indicate that the reduced pressure is kept nearly constant with the mass and the potential strength ratios, with a deviation not larger than $5 \%$ from the pure methane value. The atomic diameter influence is somewhat larger exhibiting an increase up to $15 \%$ of the pure methane value.

On the other hand, as expected, figures (1) to (3) show that a dynamic property, the thermal conductivity, is more sensitive to the molecular parameters ratios [11]. This dependency is weak for the potential strength, non negligible for the atomic diameter (up to an increase of 13\%) and becomes important for large mass ratios. Nevertheless for the moderate ratios, $\mathrm{m}_{1} / \mathrm{m}_{2}<30$ and $\sigma_{1} / \sigma_{2}<3$, the discrepancy with the pure methane value does not exceed $10 \%$.

Elsewhere, we have shown [16] that a modified version of the van der Waals one fluid approximation [22] can reduced these discrepancies on pressure but does not improve significantly the result for the thermal conductivity.

Assuming that on this range of parameters ratios the law of the corresponding states is valid for the thermal diffusion process, we have defined the pertinent variables to define a reduced thermal diffusion factor for each molecular parameters [9].

These relations being:

$$
\begin{gathered}
\alpha_{T m}^{*}=\alpha_{T}\left(\frac{m_{1}+m_{2}}{m_{1}-m_{2}}\right), m_{1} \neq m_{2} \\
\alpha_{T \varepsilon}^{*}=\alpha_{T}\left(\frac{\varepsilon_{2}}{\varepsilon_{1}-\varepsilon_{2}}\right), \varepsilon_{1} \neq \varepsilon_{2}
\end{gathered}
$$




$$
\alpha_{T \sigma}^{*}=\alpha_{T}\left(\frac{\sigma_{12}^{3}}{\sigma_{1}^{3}-\sigma_{2}^{3}}\right), \sigma_{1} \neq \sigma_{2}
$$

Figures (4) to (6) show the variation of these reduced thermal diffusion factors versus the corresponding molecular parameters ratios.

These figures indicate that, on the range of validity of the law of the corresponding states, the reduced thermal diffusion factors are correctly defined by relations (11) to (13). Elsewhere, the deviations are similar to those of the thermal conductivities which is consistent with the nature of thermal diffusion.

We have then evaluated these various reduced thermal diffusion factors which are, on average for the valid range of ratios: $\alpha_{T m}^{*}=1.55, \alpha_{T \varepsilon}^{*}=0.5235$ and $\alpha_{T \sigma}^{*}=-0.15$. The $\alpha_{T m}^{*}$ value is larger than those in dilute gases [18] but is smaller than those in liquids $[23,24]$, which is consistent with the chosen thermodynamics state.

\subsection{Coupled influence in equimolar mixtures}

As we have extracted the pertinent variables for each molecular parameter to "insert" the thermal diffusion factor in the scheme of the law of the corresponding states, the following step is to evaluate the way they combine each other.

We have first supposed that a simple sum between the contributions defined by relations (11) to (15) could be a sufficient approximation to estimate the thermal diffusion factors [9]. However, such an assumption, which implies that no couplings occur between the various molecular parameters, is a crude one. We have shown that this approximation is consistent with the law of the corresponding states only applied on thermal diffusion only when the molecular ratios are close to one $[9,13]$. Therefore we have defined a modified relation in order to take into account the couplings: 


$$
\begin{aligned}
& \alpha_{T}=\alpha_{T m}^{*} \frac{m_{1}-m_{2}}{m_{1}+m_{2}}+\alpha_{T \varepsilon}^{*} \frac{\varepsilon_{1}-\varepsilon_{2}}{\varepsilon_{2}}+\alpha_{T \sigma}^{*} \frac{\sigma_{1}^{3}-\sigma_{2}^{3}}{\sigma_{12}^{3}}+\alpha_{T m \varepsilon}^{*} \frac{m_{1}-m_{2}}{m_{1}+m_{2}}\left(\frac{\varepsilon_{1}-\varepsilon_{2}}{\varepsilon_{2}}\right) \\
& +\alpha_{T m \sigma}^{*} \frac{m_{1}-m_{2}}{m_{1}+m_{2}}\left(\frac{\sigma_{1}^{3}-\sigma_{2}^{3}}{\sigma_{12}^{3}}\right)+\alpha_{T \varepsilon \sigma}^{*} \frac{\varepsilon_{1}-\varepsilon_{2}}{\varepsilon_{2}}\left(\frac{\sigma_{1}^{3}-\sigma_{2}^{3}}{\sigma_{12}^{3}}\right)
\end{aligned}
$$

where all the coefficients appearing in this equation are function of the reduced thermodynamics state only.

We have then defined a global reduced thermal diffusion factor,

$$
\alpha_{T m \varepsilon \sigma}^{*}=\alpha_{T m}^{*} \alpha_{T \varepsilon}^{*} \alpha_{T \sigma}^{*} \alpha_{T m \varepsilon}^{*} \alpha_{T m \sigma}^{*} \alpha_{T \varepsilon \sigma}^{*}
$$

which takes into account the various contributions and can be rewritten using (14) as:

$$
\alpha_{T m \varepsilon \sigma}^{*}=\alpha_{T} \cdot\left[\begin{array}{l}
\left.\frac{1}{a}\left(\frac{m_{1}-m_{2}}{m_{1}+m_{2}}\right)+\frac{1}{b}\left(\frac{\varepsilon_{1}-\varepsilon_{2}}{\varepsilon_{2}}\right)+\frac{1}{c}\left(\frac{\sigma_{1}^{3}-\sigma_{2}^{3}}{\sigma_{12}^{3}}\right)+\frac{1}{d}\left(\frac{m_{1}-m_{2}}{m_{1}+m_{2}}\right)\left(\frac{\varepsilon_{1}-\varepsilon_{2}}{\varepsilon_{2}}\right)\right]^{-1} \\
+\frac{1}{e}\left(\frac{m_{1}-m_{2}}{m_{1}+m_{2}}\right)\left(\frac{\sigma_{1}^{3}-\sigma_{2}^{3}}{\sigma_{12}^{3}}\right)+\frac{1}{f}\left(\frac{\varepsilon_{1}-\varepsilon_{2}}{\varepsilon_{2}}\right)\left(\frac{\sigma_{1}^{3}-\sigma_{2}^{3}}{\sigma_{12}^{3}}\right)
\end{array}\right]^{3}
$$

where

$$
\begin{gathered}
a=\alpha_{T \varepsilon}^{*} \alpha_{T \sigma}^{*} \alpha_{T m \varepsilon}^{*} \alpha_{T m \sigma}^{*} \alpha_{T \varepsilon \sigma}^{*}, b=\alpha_{T m}^{*} \alpha_{T \sigma}^{*} \alpha_{T m \varepsilon}^{*} \alpha_{T m \sigma}^{*} \alpha_{T \varepsilon \sigma}^{*}, \\
c=\alpha_{T m}^{*} \alpha_{T \varepsilon}^{*} \alpha_{T m \varepsilon}^{*} \alpha_{T m \sigma}^{*} \alpha_{T \varepsilon \sigma}^{*}, d=\alpha_{T m}^{*} \alpha_{T \varepsilon}^{*} \alpha_{T \sigma}^{*} \alpha_{T m \sigma}^{*} \alpha_{T \varepsilon \sigma}^{*} \\
e=\alpha_{T m}^{*} \alpha_{T \varepsilon}^{*} \alpha_{T \sigma}^{*} \alpha_{T m \varepsilon}^{*} \alpha_{T \varepsilon \sigma}^{*}, f=\alpha_{T m}^{*} \alpha_{T \varepsilon}^{*} \alpha_{T \sigma}^{*} \alpha_{T m \varepsilon}^{*} \alpha_{T m \sigma}^{*}
\end{gathered}
$$

We have evaluated the coefficients characterizing the couplings, $\alpha_{T m \varepsilon}^{*}, \alpha_{T m \sigma}^{*}$ and $\alpha_{T \varepsilon \sigma}^{*}$ thanks to separate simulations performed on synthetic binary mixtures having two molecular ratios different from one at the same time $[9,16]$. We have found on average $\alpha_{T m \varepsilon}^{*}=0.25 \alpha_{T m \sigma}^{*}=-0.35$ and $\alpha_{T \varepsilon \sigma}^{*}=-0.15$ which implies, using (15), that:

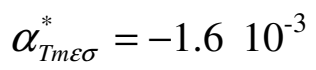

In order to test the applicability of equation (16) in the corresponding state formalism, we have performed simulations on various equimolar mixtures where the second 
component is always methane. The molecular parameters of the involved components, except methane and $n$-decane, have been taken from [25].

For all the mixtures tested, the validity of the computed reduced thermal conductivities and pressures do not deviate more than $10 \%$ from the pure methane value.

Results given in table (1) indicate that the reduced thermal diffusion factor in equimolar mixtures defined by relation (16) is nearly constant whatever the mixture. Moreover, the values are close to the one obtained in relation (18), the relative deviation being $7.5 \%$ at most.

\subsection{Alkanes equimolar mixtures}

To confirm the first results obtained in the previous section, we have simulated various $n$-alkane/methane and $n$-alkane/ethane mixtures. These mixtures are of interest to test the quality of the vdW1 approximation for petroleum mixtures. The molecular parameters of the various normal alkanes have been taken from [19]. It should be noted that for the biggest alkanes tested, the LJ sphere approach is a poor approximation of the real component.

For $n$-alkane/methane mixtures, figure (7) shows that the deviations of the two reduced properties from the pure methane value are reasonable; the discrepancy goes up to $10 \%$ on the pressure and up to $20 \%$ for the thermal conductivity. The difference increases with the size of the $n$-alkane studied. It is interesting to notice that, for these realistic mixtures, this is the dynamic property which departs the most from the pure component value as expected [11]. Moreover, figure (7) is similar to figure (1), which indicates that the mass contribution is the one that induces the major part of the deviation from the law of the corresponding states. Nevertheless, it seems that the vdW1 approximation is sufficient, at least up to the hexadecane, to fulfil the law the corresponding states in the cases studied. 
Figure (8) shows that the reduced thermal diffusion factor defined by equation (16) fluctuates slightly with the carbon number of the $n$-alkane involved. Nevertheless, this figure indicates that $\alpha_{T m \varepsilon \sigma}^{*}$ deduced from computations is always close to the value given by equation (18), meaning that the way we defined the reduced thermal diffusion factor is consistent for such mixtures. More precisely, starting from relation (14), the estimated thermal diffusion factor in these mixtures does not deviate more than $15 \%$ from the computed value.

As the parameters appearing in relation (16) have been evaluated using methane as the reference component, we have tested this relation using ethane as the reference species. The simulations on $n$-alkane/ethane have been performed in the same reduced thermodynamics state. For the tested mixtures, the reduced pressures and thermal conductivities do not deviate more than $8 \%$ from the pure component values.

Figure (9) indicates that that the reduced thermal diffusion factor is still correctly defined when changing the reference component, because its value is kept nearly constant whatever the mixture and is close to the one given by equation (18). When relation (14) is applied for these mixtures, the estimated thermal diffusion factor does not exhibit a discrepancy larger than $15 \%$ from the computed value. However, for these ethane/n-alkane mixtures, the estimated thermal diffusion factor is always lower than the computed one.

\subsection{Results in nonequimolar binary mixtures}

In the previous sections only equimolar mixtures have been evaluated, but when dealing with thermal diffusion, the relative proportion of the two involved components is a crucial parameter as well.

In a previous work, we have shown, for another reduced thermodynamics state, the possibility to estimate the thermal diffusion factor in nonequimolar mixtures using a 
simple correlation which needs both infinite dilution thermal diffusion factors and molar fractions [17]. For alkane mixtures it is possible to link linearly the thermal diffusion factor to the mass fraction:

$$
\alpha_{T}=\left(\alpha_{T}\right)_{w_{2}=0}+\left(\left(\alpha_{T}\right)_{w_{1}=0}-\left(\alpha_{T}\right)_{w_{2}=0}\right) w_{2}
$$

where the $\left(\alpha_{T}\right)_{w_{i}=0}$ are the infinite dilution thermal diffusion factors and $w_{2}$ the methane mass fraction.

Moreover, we have found that for a large variety of alkane mixtures we can relate these infinite dilution factors to the ratio of the molar masses:

$$
\frac{\left(\alpha_{T}\right)_{w_{1}=0}}{\left(\alpha_{T}\right)_{w_{2}=0}}=\frac{m_{1}}{m_{2}}
$$

Then, combining equations (19) and (20), we can express the thermal diffusion factor in function of only one infinite dilution value:

$$
\alpha_{T}=\left(\alpha_{T}\right)_{w_{2}=0}\left(1+\frac{m_{1}-m_{2}}{m_{2}} w_{2}\right)
$$

If we now consider the equimolar case, as in the previous sections, relation (21) enables us to link an infinite dilution value to the equimolar one in the following way:

$$
\left(\alpha_{T}\right)_{w_{2}=0}=\frac{m_{1}+m_{2}}{2 m_{1}}\left(\alpha_{T}\right)_{x_{2}=1 / 2}
$$

Reinserting this relation in equation (21) we obtain:

$$
\left(\alpha_{T}\right)_{x_{2}=1 / 2}=\frac{\alpha_{T}}{\frac{m_{1}+m_{2}}{2 m_{1}}\left(1+\frac{m_{1}-m_{2}}{m_{2}} w_{2}\right)}
$$

It is then possible, in the spirit of equation (8), to deduce a comprehensive reduced thermal diffusion factor that takes into account the influence of all the contributions evaluated by combining equation (23) and equation (16): 


$$
\alpha_{T}^{*}=\frac{\alpha_{T}}{z_{\alpha_{T}}\left(m_{1}, m_{2}, \varepsilon_{1}, \varepsilon_{2}, \sigma_{1}, \sigma_{2}, w_{2}\right)}
$$

where

$$
\begin{aligned}
& z_{\alpha_{T}}\left(m_{i}, \varepsilon_{i}, \sigma_{i,} w_{i}\right)_{i=1,2}=\left[\frac{m_{1}+m_{2}}{2 m_{1}}\left(1+\frac{m_{1}-m_{2}}{m_{2}} w_{2}\right)\right] \times \\
& {\left[\begin{array}{l}
\left.\frac{1}{a}\left(\frac{m_{1}-m_{2}}{m_{1}+m_{2}}\right)+\frac{1}{b}\left(\frac{\varepsilon_{1}-\varepsilon_{2}}{\varepsilon_{2}}\right)+\frac{1}{c}\left(\frac{\sigma_{1}^{3}-\sigma_{2}^{3}}{\sigma_{12}^{3}}\right)+\frac{1}{d}\left(\frac{m_{1}-m_{2}}{m_{1}+m_{2}}\right)\left(\frac{\varepsilon_{1}-\varepsilon_{2}}{\varepsilon_{2}}\right)\right] \\
+\frac{1}{e}\left(\frac{m_{1}-m_{2}}{m_{1}+m_{2}}\right)\left(\frac{\sigma_{1}^{3}-\sigma_{2}^{3}}{\sigma_{12}^{3}}\right)+\frac{1}{f}\left(\frac{\varepsilon_{1}-\varepsilon_{2}}{\varepsilon_{2}}\right)\left(\frac{\sigma_{1}^{3}-\sigma_{2}^{3}}{\sigma_{12}^{3}}\right)
\end{array}\right]}
\end{aligned}
$$

To apply equation (24) to estimate the thermal diffusion factor in a mixture at $T^{*}=2.273$ and $\rho^{*}=0.4227$, we have supposed that the value defined in equilibrium mixture is a good approximation, meaning that:

$$
\alpha_{T}^{*}=-1.610^{-3}
$$

We have performed simulations on various nonequimolar mixtures of $n$-decane/methane in the same reduced thermodynamics state than previously, the methane being always for sign convenience component 2 . The validity of the vdW1 approximation in non equimolar mixtures is of primary importance because it will enable us to estimate the transport properties of multicomponents mixtures. We have carried on simulations on mixtures where the molar fraction of methane goes from 0.02 to 0.98 , larger dilution being hard to achieve through molecular dynamics simulation due to the weakness of the statistic. The statistical errors on the computed thermal diffusion factors go up to 15 $\%$ for the largest dilution.

Figure (10) obviously indicates that the reduced thermophysical properties tested are kept nearly constant for the whole range of molar fraction computed, meaning that the vdW1 approximation is efficient concerning the molar fraction variable. The reduced 
pressure never exceeds $10 \%$ of deviation from the pure methane value, and the reduced thermal conductivity exhibits a reduction of at most $5 \%$ from the reference value.

Figure (11) shows that for this particular mixture the reduced thermal diffusion factor is weakly dependent to the molar fractions, the variation being of the order of the statistical errors. This result means that the reduced thermal diffusion factor is correctly defined by relation (24). Moreover, on average, $\alpha_{T}^{*}=-1.5510^{-3}$, which is a value close to the one deduced from the systematic study given by equation (26).

So, for this particular mixture, when relation (24) is used in reverse, we are able to estimate the thermal diffusion factor within the error bars, with a deviation of $6.5 \%$ at most, starting only from the molecular parameters of the two components.

In order to assess the validity of relation (24) for a wider range of alkanes LJ mixtures, we have carried on simulations on $n$-pentane/methane and $n$-decane $/ n$-pentane mixtures. The simulations have been performed for the same range of molar fraction than for the methane/n-decane mixture.

Figure (12) shows that reduced thermal diffusion factors deduced from computations and relation (24) do not exhibit a strong dependence to the $n$-pentane molar fraction for the two mixtures studied. Nevertheless slight differences, from a constant value, appear when one species is sufficiently predominant. This trend could be understood as the statistical error bars increase with the dilution.

Elsewhere, on average, $\alpha_{T}^{*}=-1.6310^{-3}$ for the $n$-pentane/methane mixture and $\alpha_{T}^{*}=-1.6110^{-3}$ for the $n$-decane/n-pentane one. These two values are close to the one given by equation (26) which means that combining equations (26) and (24) yields a good estimation of the thermal diffusion factors of these two LJ mixtures. More precisely, using these two relations allow us to estimate $\alpha_{T}$ with a precision better than 
$8 \%$ for the $n$-pentane/methane mixture and $13 \%$ for the $n$-decane $/ n$-pentane one for all the points simulated.

\subsection{Results in ternary mixtures}

Petroleum fluids involve multicomponents mixtures, so, in order to provide a first step towards the comprehension of such mixtures, we have performed simulations on ternary alkane mixtures.

The aim of this part of the study is to show the possibility to estimate LJ thermal diffusion factors in ternary mixtures using the correlation developed, equation (24), and the van der Waals one fluid approximation.

In order to do so, we have carried on simulations in the same reduced thermodynamics state as previously. The two involved ternary mixtures are a methane/ethane/n-decane one and a methane $/ n$-pentane $/ n$-decane one. The molar fraction of methane, for the two mixtures, varied from 0.07 to 0.93 . The molar fraction of the two others species have been taken equal to each other for simplicity.

To use relation (24), which has been constructed for binary mixture, we have defined "equivalent" binary mixtures to mimic the behaviour of the ternary ones. These "equivalent" binary mixtures are composed of methane and of a pseudocomponent representing the two other ones. The pseudocomponents have been defined thanks to the van der Waals one fluid approximation, relations (6), (7) and (9), applied on ethane/ $n$ decane and on $n$-pentane/ $n$-decane species. In a previous work [17] we have shown that an "equivalent" binary mixture defined so correctly mimics the thermal diffusion behaviour of the ternary mixture represented.

For the methane/ethane/n-decane mixtures, figure (13) shows that the reduced pressure and the reduced thermal conductivity follow correctly the law of the corresponding state when changing the methane molar fraction. Moreover the average values are close to 
the pure methane one indicating that the vdW1 is efficient for this ternary mixture. Nevertheless, figure (13) shows a reduction up to $9 \%$ of the reduced thermal conductivity compared to the pure component value. Once again it appears that the dynamic property deviates more than the static one from the law of the corresponding state.

Concerning the reduced methane thermal diffusion factors deduced from the simulation and the use of relation (24) applied on binary "equivalent" mixtures, their values are positive contrary to previously as shown by figure (14). This comes from the fact that the reference component, the methane, is defined as component 1 in these ternary mixtures whereas for the previous cases the reference species was defined as component 2.

Figure (14) indicates that the reduced methane thermal diffusion factors for the two ternary mixtures are weakly dependent to the methane molar fraction. This result means that, even for ternary mixtures, relation (24) is consistent when a one fluid approximation for component 2 and 3 is used. Moreover, on average, $\alpha_{\text {Tmeth }}^{*}=1.5310^{-3}$ in the methane/ethane/n-decane mixture and $\alpha_{\text {Tmeth }}^{*}=1.5610^{-3}$ in the methane $/ n$ pentane $/ n$-decane one, which is a consistent value compared to the one given by equation (26), apart from the sign. Starting from relation (24) combined with a vdW1 approximation it is thus possible to estimate the methane $\mathrm{LJ}$ thermal diffusion factors in these two ternary mixtures with accuracy better than $10 \%$.

So, the definition of the reduced thermal diffusion factor through relation (24) yields consistent results for a large variety of Lennard-Jones mixtures. Nevertheless further simulations should be carried on to assess the validity of such relation for different thermodynamics states.

\section{Conclusion}


We have shown that NonEquilibrium Molecular Dynamics, applied on simple LennardJones spheres, is an efficient tool to estimate the pertinent parameters that induce thermal diffusion in fluid mixtures for a given supercritical thermodynamics state. The van der Waals one fluid approximation has been applied and has shown to fulfil the principle of the corresponding states quite correctly for a wide variety of molecular parameters and molar fractions when applied to pressure and thermal conductivity. In this frame, we have shown that it is possible to find the relevant variables that enable us to formulate a physically based reduced thermal diffusion factor. This coefficient takes into account each molecular contribution, namely the mass, the atomic diameter, the potential strength and the mass fraction. Using this reduced thermal diffusion factor, which encompasses the couplings between the molecular contributions, allows a good estimation of thermal diffusion of LJ mixtures compared to molecular dynamics simulations. Finally, this scheme has shown to yield quite accurate thermal diffusion factor values of alkane binary and ternary LJ mixtures starting only from the molecular parameters of the mixture, the deviations from the computed values being always lower than $15 \%$ for all points simulated.

\section{List of symbols}

$\begin{array}{ll}D_{12} & \text { mutual diffusion coefficient, } \mathrm{m}^{2} \cdot \mathrm{s}^{-1} \\ D_{T} & \text { thermal diffusion coefficient, } \mathrm{m}^{2} \cdot \mathrm{s}^{-1} \cdot \mathrm{K}^{-1} \\ J_{m} & \text { mass flux, } \mathrm{kg} \cdot \mathrm{m}^{-2} \cdot \mathrm{s}^{-1} \\ k_{b} & \text { Boltzmann constant, } \mathrm{J} \cdot \mathrm{K}^{-1} \\ m & \text { molar mass, } \mathrm{kg} \cdot \mathrm{mol}^{-1} \\ N & \text { number of molecules } \\ P & \text { pressure, } \mathrm{Pa}\end{array}$




$$
\begin{array}{ll}
r & \text { distance between two molecules, } \mathrm{m} \\
T & \text { temperature, } \mathrm{K} \\
U_{L J} & \text { Lennard-Jones potential energy, } \mathrm{J} \\
V & \text { volume, } \mathrm{m}^{3} \\
w & \text { mass fraction } \\
x & \text { molar fraction } \\
\mathrm{Z} & \text { transport characteristic function }
\end{array}
$$

Greek symbols:

$$
\begin{array}{ll}
\alpha_{T} & \text { thermal diffusion factor } \\
\varepsilon & \text { potential depth, } \mathrm{J} \\
\kappa & \text { thermal conductivity, } \mathrm{W} \cdot \mathrm{K}^{-1} \cdot \mathrm{m}^{-1} \\
\rho & \text { density, } \mathrm{kg} \cdot \mathrm{m}^{-3} \\
\sigma & \text { molecular diameter, } \mathrm{m}
\end{array}
$$

Subscripts:

$$
\begin{array}{ll}
c & \text { critical value } \\
i, j & \text { component number } \\
x & \text { characteristic quantity of the mixture }
\end{array}
$$

Superscripts:

* $\quad$ reduced quantity

\section{Acknowledgement}

This research was supported by TOTAL. I am grateful to F. Montel who has initiated and improved the work done by fruitful advices and comments. I would like to thank as well, B. Duguay and J.-P. Caltagirone for profitable discussions. This work has been 
made possible thanks to generous grants of computing time by the CINES, IDRIS and SIMOA.

\section{References}

[1] Faissat B., Montel F., Place de la thermodiffusion dans l'évaluation de l'état initial des gisements pétroliers, Entropie 198/199 (1996) 107-108.

[2] Hafskjold B., Ikeshoji T., Ratkje S.K., On the molecular mechanism of thermal diffusion in liquids, Mol. Phys. 80 (1993) 1389-1412.

[3] Kempers L.J.T.M, A comprehensive thermodynamics theory of the Soret effect in a multicomponent gas, liquid or solid, J. Chem. Phys. 115 (2001) 6330-6341.

[4] Firoozabadi A., Ghorayeb K., Shukla K., Theoritical model of thermal diffusion factors in multicomponent mixtures, Aiche Journal 46 (2000), 892-900.

[5] Wiegand S., Thermal diffusion in liquid mixtures and polymer solutions, J. Phys.: Condens. Matter 16 (2004), 357-379.

[6] Reith D., Müller-Plathe F., On the nature of thermal diffusion in binary LennardJones liquids, J. Chem. Phys. 112 (2000) 2436-2443.

[7] Nieto-Draghi C., Transport and structural properties of aqueous solutions of organic solvents, Phd Thesis, Universitat Rovira et Virgili, 2003.

[8] Perronace A., Leppla C., Leroy F., Rousseau B., Wiegand S., Soret and mass diffusion measurements and molecular dynamics simulation of $\mathrm{n}$-pentane/n-decane mixtures, J. Chem. Phys. 116 (2002) 3718-3729.

[9] Galliéro G., Duguay B., Caltagirone J.-P., Montel F., Thermal diffusion sensitivity to the molecular parameters of a binary equimolar mixture, a nonequilibrium molecular dynamics approach, Fluid Phase Equi. 208 (2003) 171-188. 
[10] Vidal J., Thermodynamique: application au génie chimique et à l'industrie pétrolière, Technip, Paris, 1997.

[11] Boon J.P., Yip S., Molecular hydrodynamics, Dover, New York, 1991.

[12] Hansen J.P., McDonald I.R., Theory of simple liquids, Academic Press, London, 1986.

[13] Galliéro G., Duguay B., Caltagirone J.-P., Montel F., De l'influence sur la thermodiffusion des paramètres d'un mélange binaire de sphères de Lennard-Jones par dynamique moléculaire hors équilibre, Entropie 239/240 (2002) 93-97.

[14] de Groot S.R., Mazur P., Nonequilibrium thermodynamics, Dover, New York, 1984.

[15] Rapaport D.C., The art of molecular dynamics, Cambridge press, Cambridge, 1995.

[16] Galliéro G., Thermodiffusion dans les fluides de Lennard-Jones par dynamique moléculaire, Phd Thesis, University of Bordeaux I, 2003.

[17] Galliéro G., Duguay B., Caltagirone J.-P., Montel F., On thermal diffusion in binary and ternary mixtures by nonequilibrium molecular dynamics, Phil. Mag. 83 (2003) 2097-1108.

[18] Chapman S., Cowling T.G., The mathematical theory of non uniform gases, Cambridge Mathematical Library, Cambridge, 1990.

[19] Sun T., Teja A. S., Vapor-liquid and solid-fluid equilibrium calculation using a Lennard-Jones Equation of State, Ind. Eng. Chem. Res. 37 (1998) 3151-3158.

[20] Simon J. M., Rousseau B., Dysthe D. K., Hafskjold B., Thermal diffusion in methane $n$-decane mixtures by molecular dynamics using spherical and flexible multicenter models, Entropie 217 (1999) 29-32. 
[21] Dysthe D.K., Fuchs A.H., Rousseau B., Prediction of fluid transport properties by equilibrium molecular dynamics, International Journal of Thermophysics 19 (1998) 437-448.

[22] Chen J., Mi J.-G., Chan K.-Y., Comparison of different mixing rules for prediction of density and residual energy of binary and ternary Lennard-Jones mixtures, Fluid Phase Equi. 178 (2000) 87-95.

[23] Debuschewitz C., Köhler W., Molecular origin of thermal diffusion in Benzene+Cyclohexane mixtures, Phys. Rev. Lett. 87 (2001) 055901-1/055901-5.

[24] Rutherford W., M. Isotopic thermal diffusion of carbon disulfide in the liquid phase, J. Chem. Phys. 86 (1987) 397-399.

[25] Hecht C. E., Statistical thermodynamics and kinetic theory, Dover, New York, 1998. 


\section{Figures and table captions:}

Figure 1: Reduced pressure $(\boldsymbol{\nabla})$ and thermal conductivity $(\boldsymbol{\Lambda})$ in supercritical binary equimolar mixtures for various mass ratios.

Figure 2: Reduced pressure $(\boldsymbol{\nabla})$ and thermal conductivity $(\boldsymbol{\Delta})$ in supercritical binary equimolar mixtures for various potential depth ratios.

Figure 3: Reduced pressure $(\boldsymbol{\nabla})$ and thermal conductivity $(\boldsymbol{\Delta})$ in supercritical binary equimolar mixtures for various atomic diameter ratios.

Figure 4: Reduced thermal diffusion factor, relation (11), in equimolar supercritical mixtures for different mass ratios.

Figure 5: Reduced thermal diffusion factor, relation (12), in equimolar supercritical mixtures for different potential depth ratios.

Figure 6: Reduced thermal diffusion factor, relation (13), in equimolar supercritical mixtures for different atomic diameter ratios.

Figure 7: Reduced pressure $(\boldsymbol{\nabla})$ and thermal conductivity $(\boldsymbol{\Delta})$ in some supercritical binary equimolar mixtures of methane/n-alkane, in function of the $n$-alkane carbon atoms number.

Figure 8: Reduced thermal diffusion factor, relation (16), in equimolar supercritical methane/n-alkane mixtures, in function of the $n$-alkane carbon atom number.

Figure 9: Reduced thermal diffusion factor, relation (16), in equimolar supercritical ethane $/ n$-alkane mixtures, in function of the $n$-alkane carbon atom number.

Figure 10: Reduced pressure $(\boldsymbol{\nabla})$ and thermal conductivity $(\boldsymbol{\Delta})$ in some supercritical binary nonequimolar mixtures of methane/n-decane, in function of the methane molar fraction.

Figure 11: Reduced thermal diffusion factor, relation (24), in nonequimolar supercritical mixtures of methane/ $n$-decane for different methane molar fractions. 
Figure 12: Reduced thermal diffusion factor in methane/n-pentane, $(\boldsymbol{\nabla})$, and ethane $/ n$ pentane, $(\boldsymbol{\Delta})$, mixtures for various pentane molar fractions.

Figure 13: Reduced pressure $(\boldsymbol{\nabla})$ and thermal conductivity $(\boldsymbol{\Delta})$ in ternary mixtures of methane/ethane/n-decane in function of the methane molar fraction.

Figure 14: Reduced methane thermal diffusion factor in methane/ethane/n-decane, $(\boldsymbol{\nabla})$, and in methane $/ n$-pentane $/ n$-decane, $(\boldsymbol{\Delta})$, mixtures for various methane molar fractions.

Table 1: Reduced thermal diffusion factors, equation (16), in various equimolar methane/simple component mixtures. 
Figure 1:

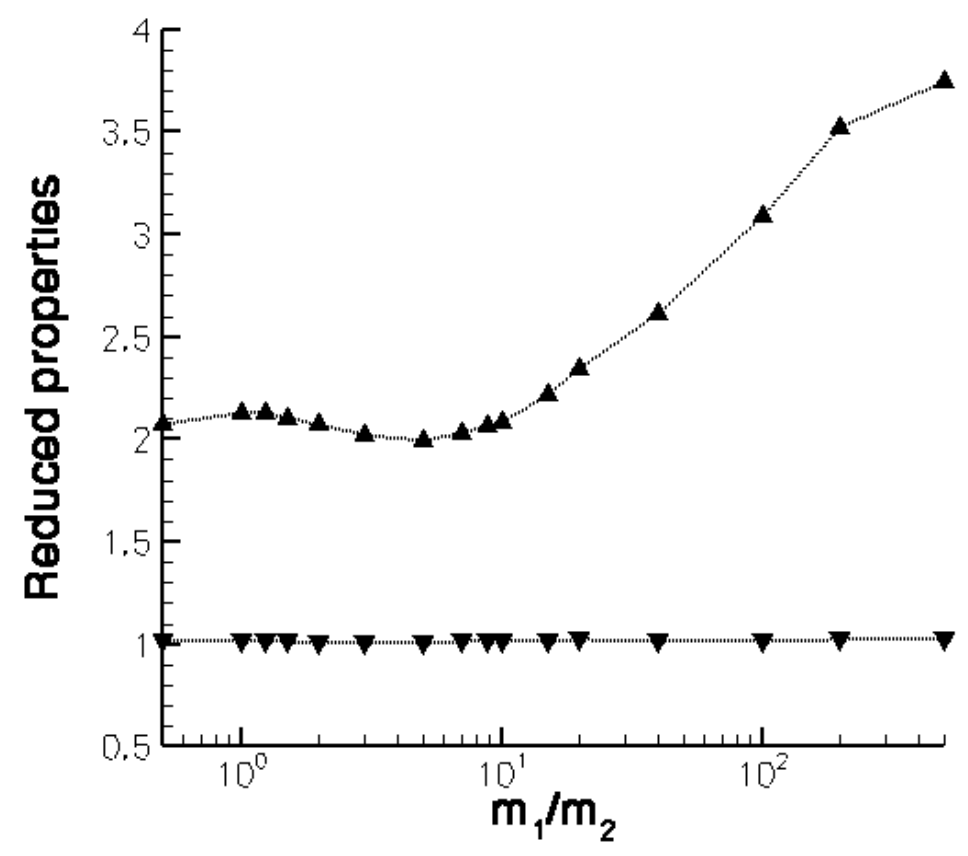

Figure 2:

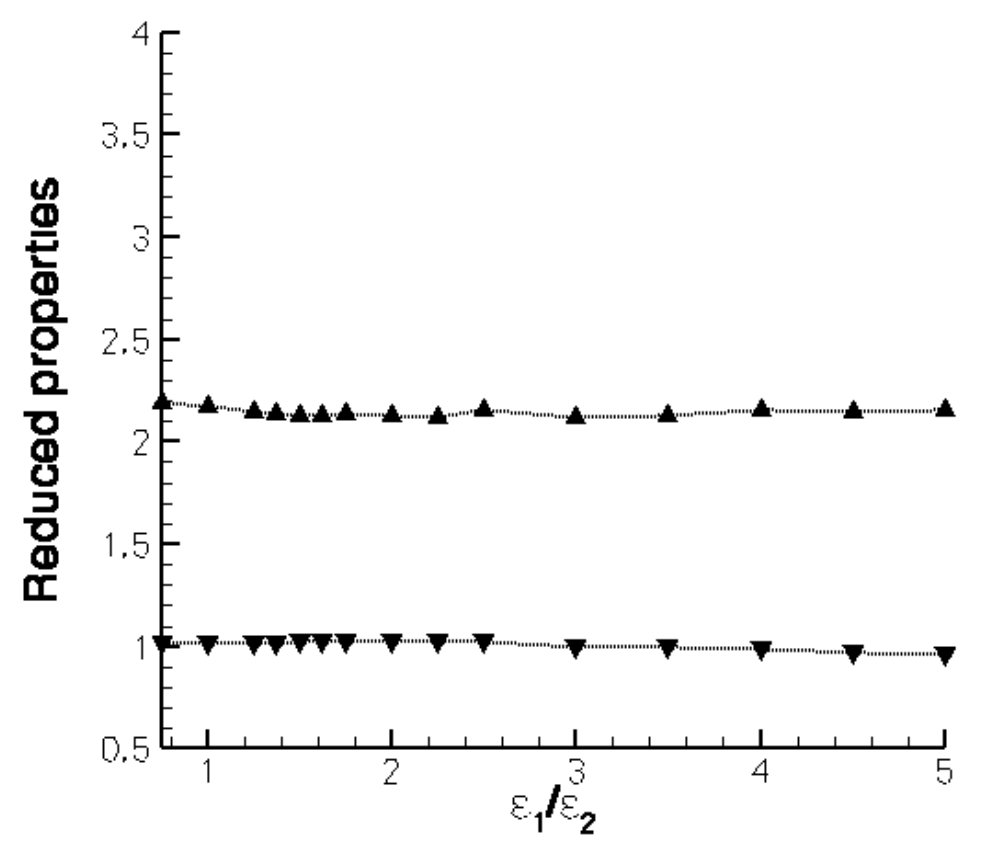


Figure 3 :

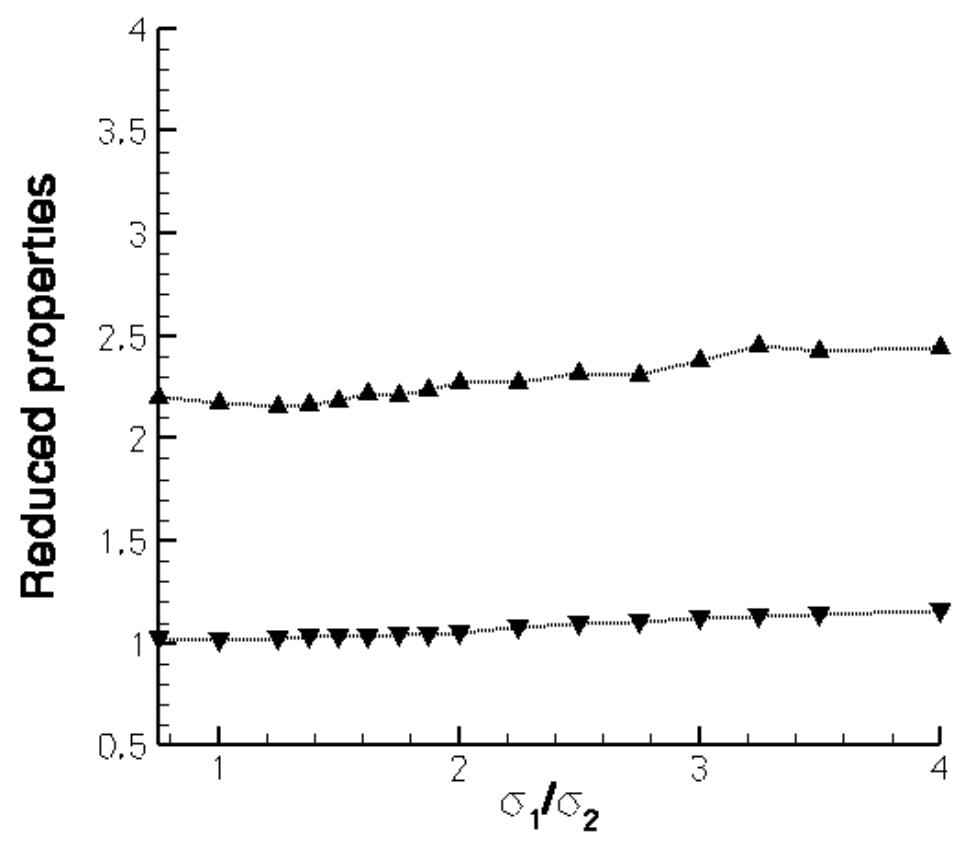

Figure 4 :

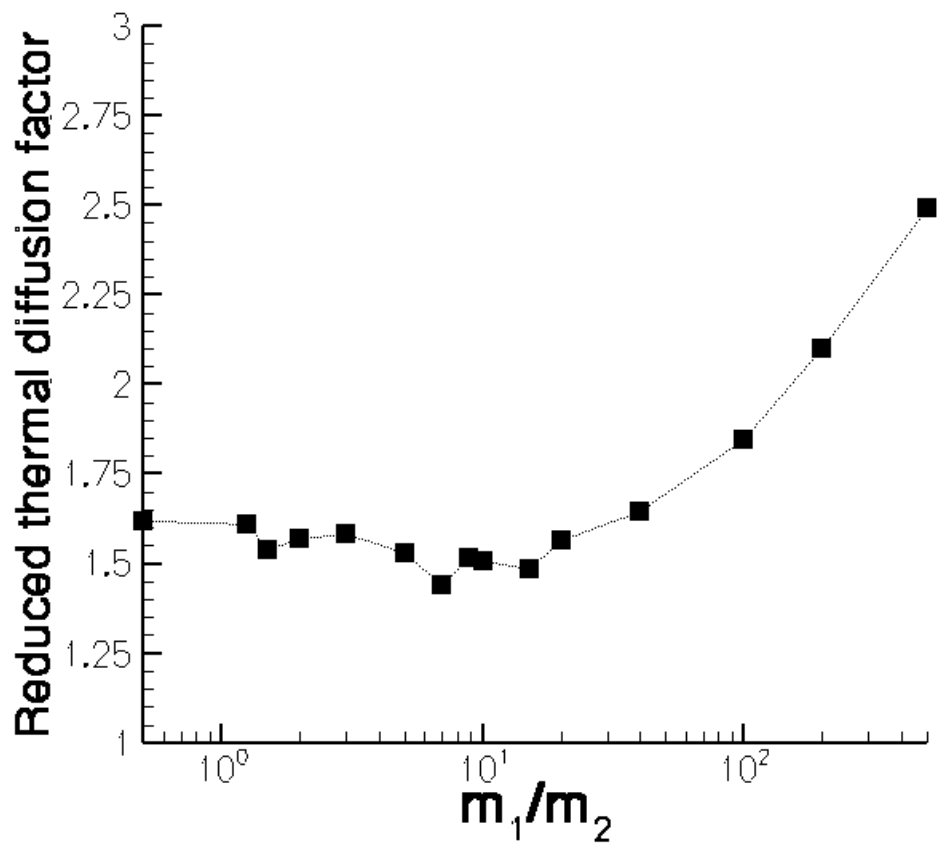


Figure 5 :

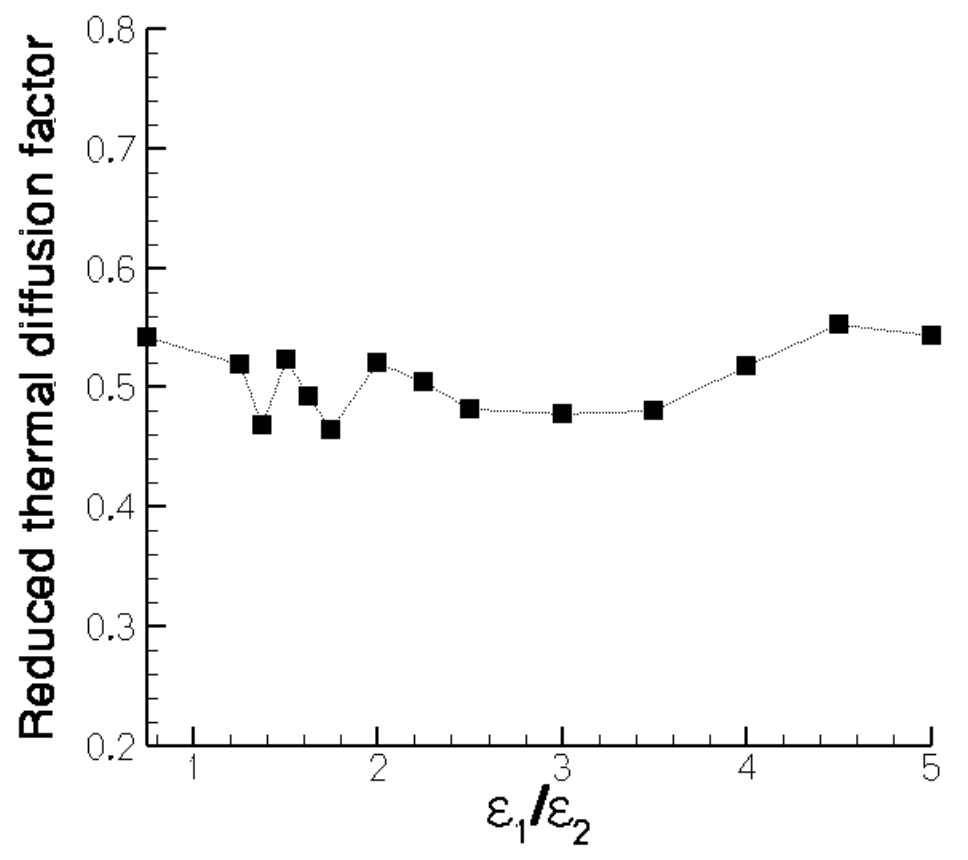

Figure 6 :

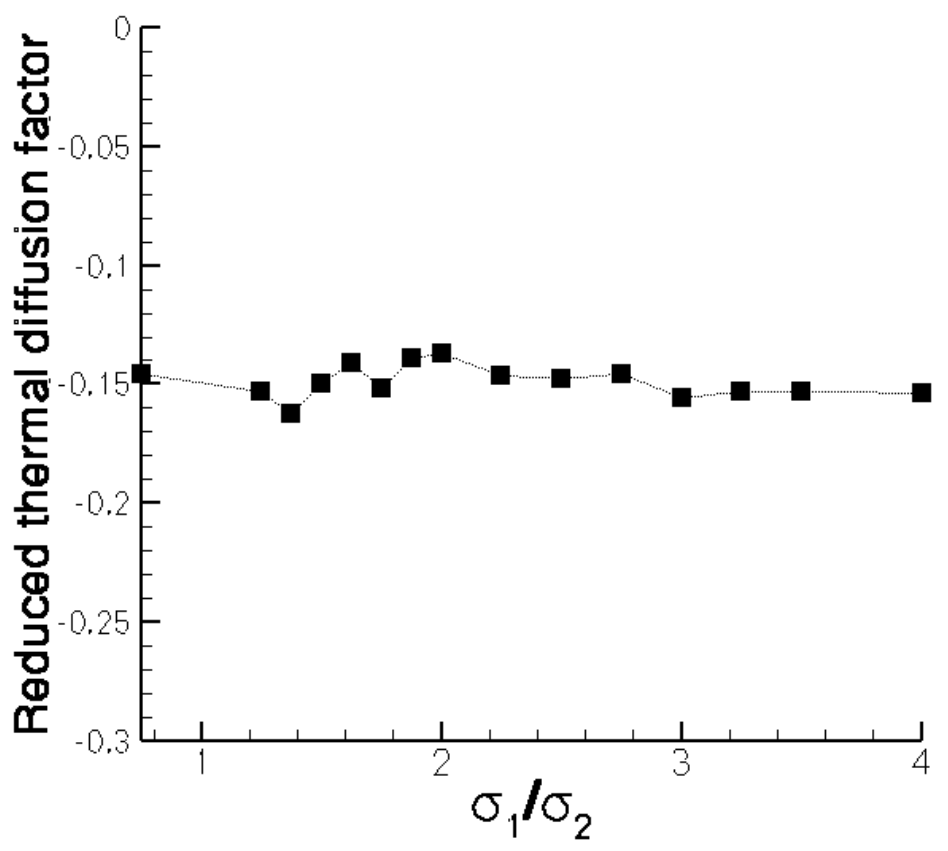


Figure 7 :

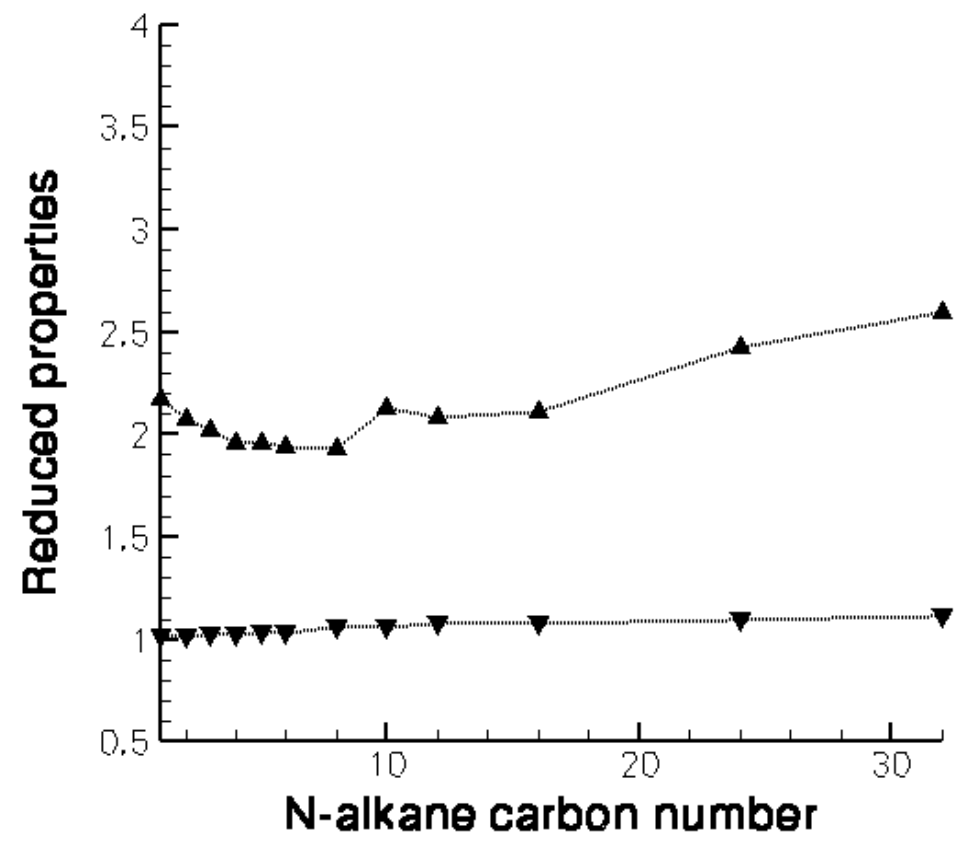

Figure 8 :

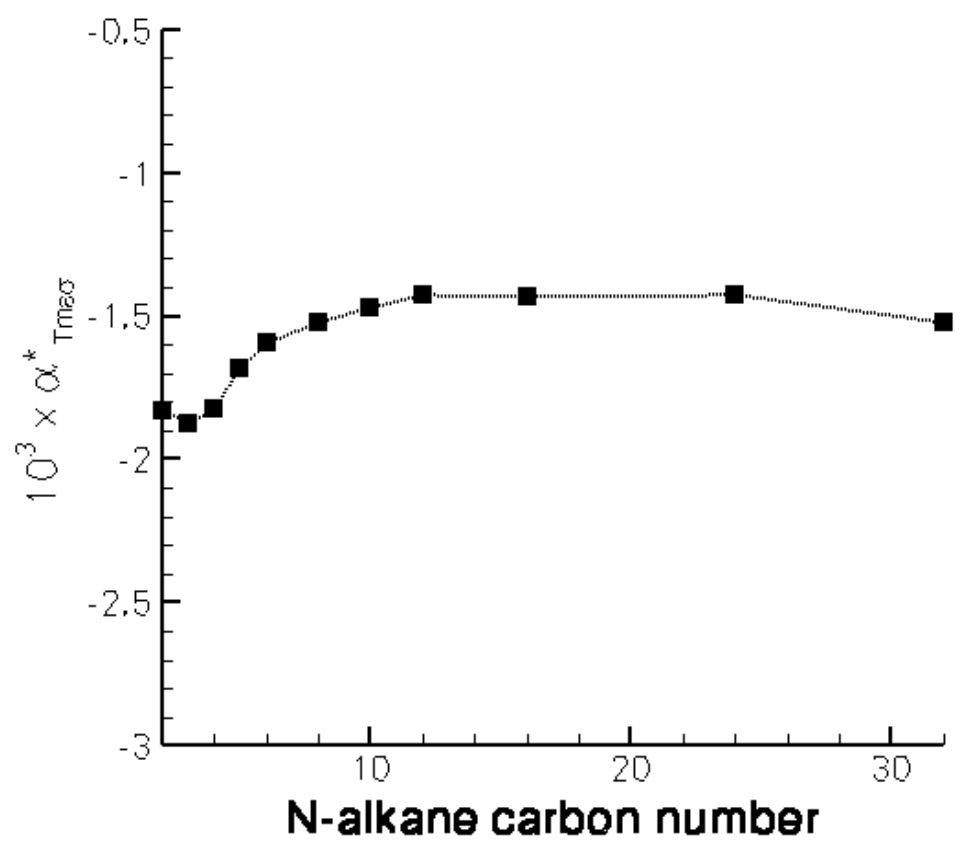


Figure 9 :

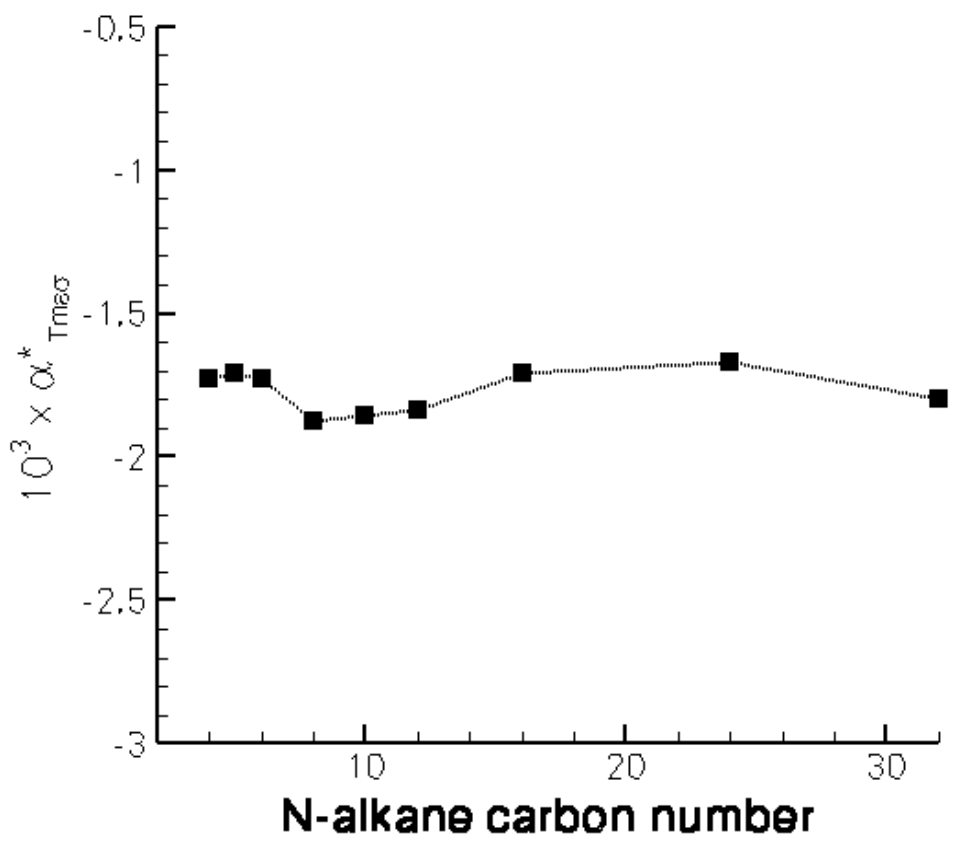

Figure 10:

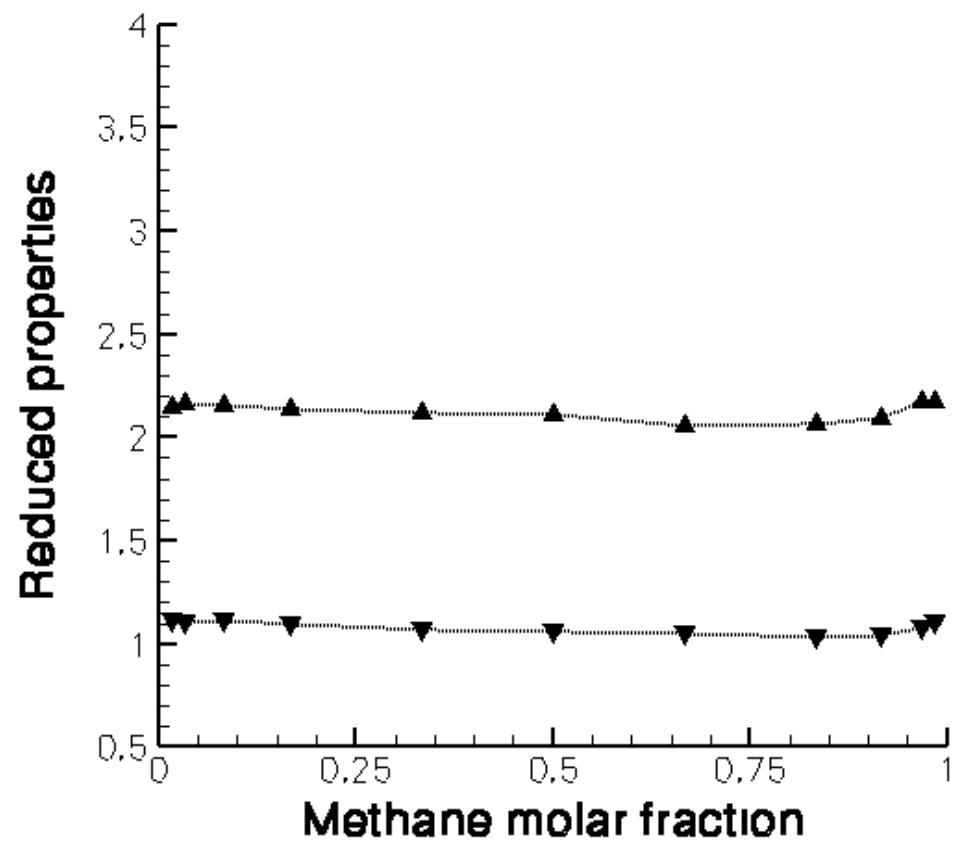


Figure 11:

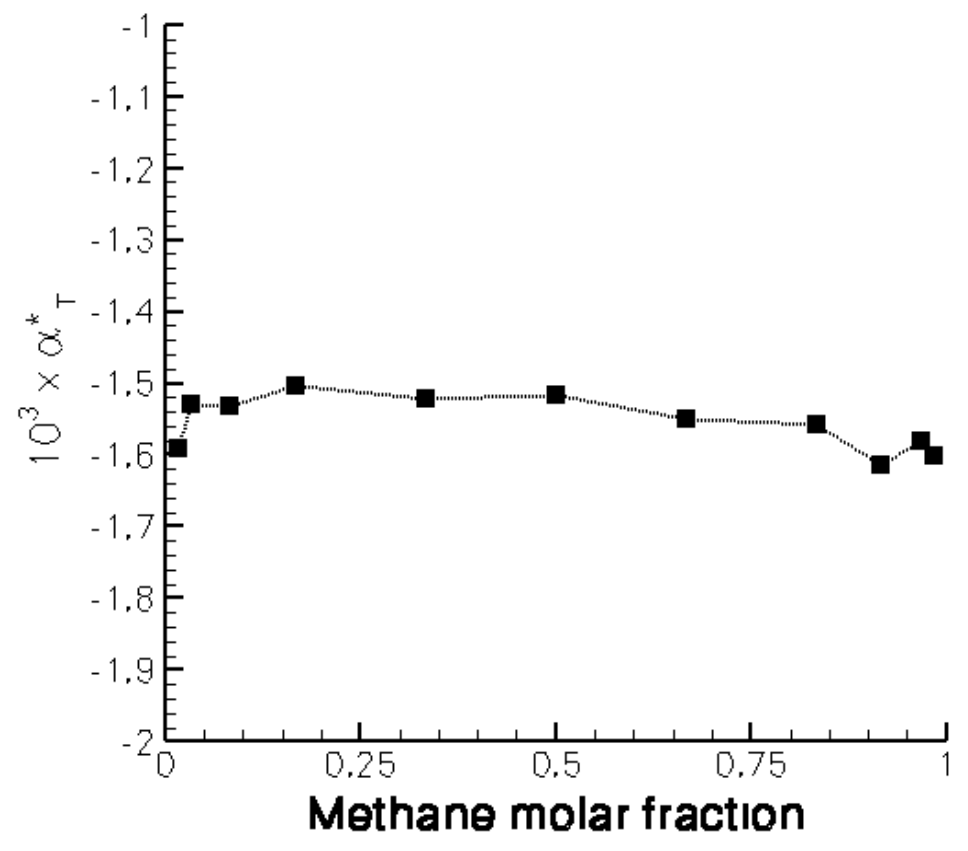

Figure 12:

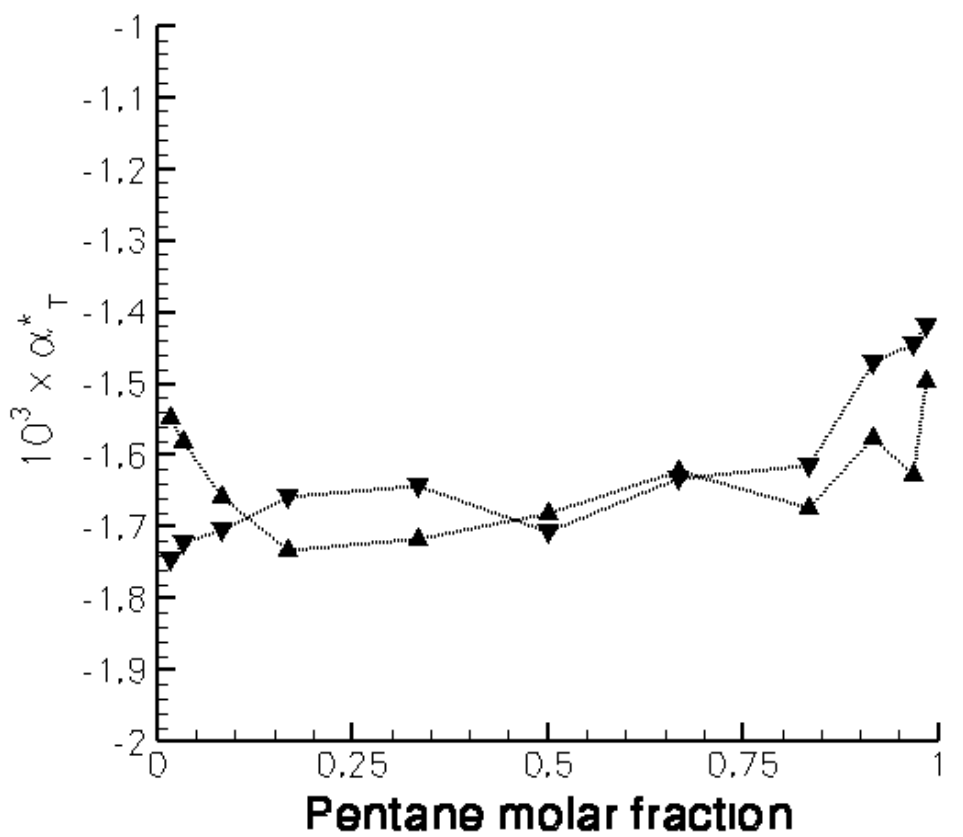


Figure 13:

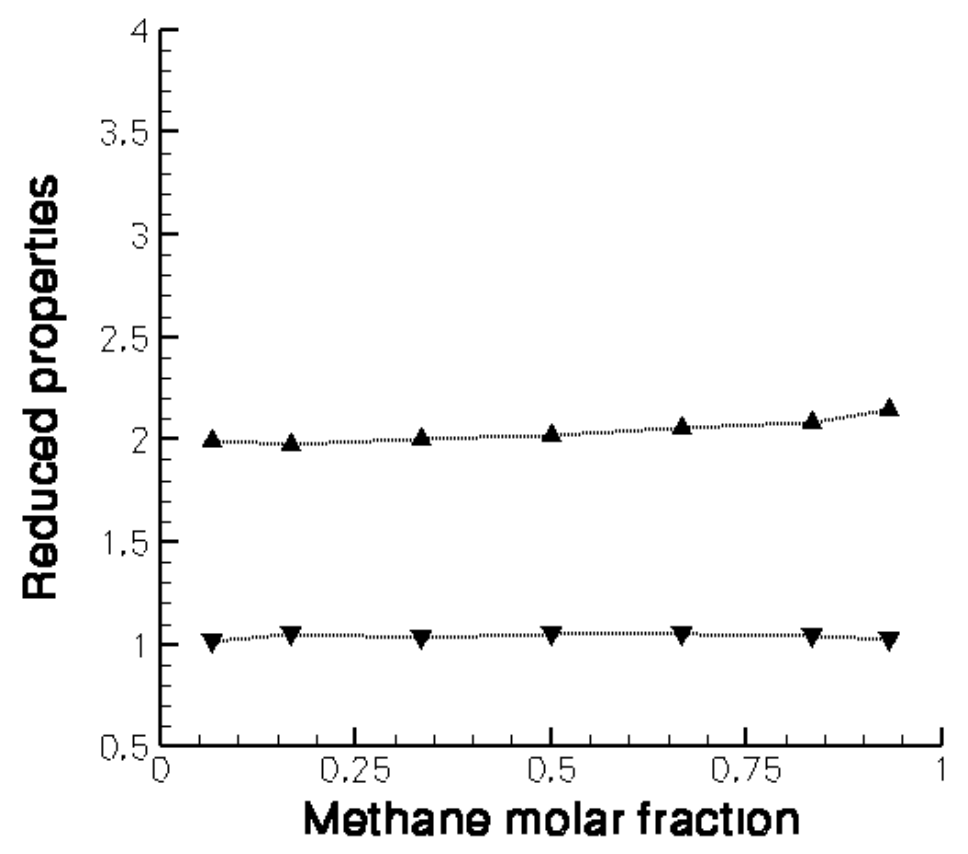

Figure 14:

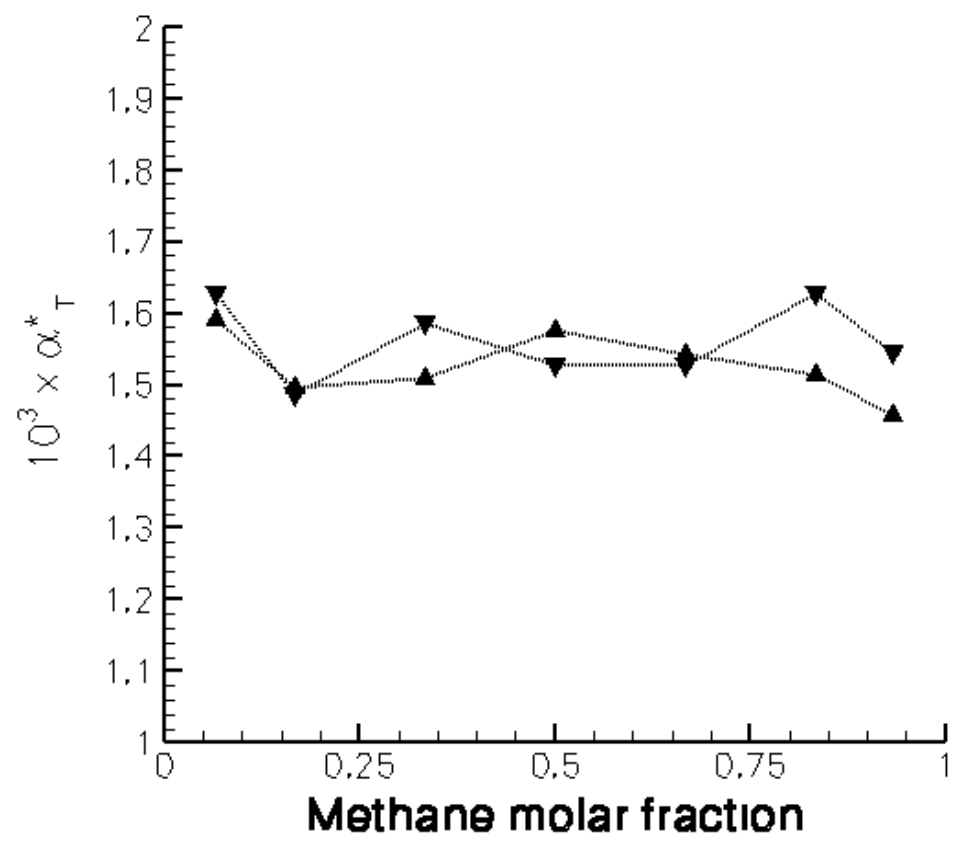


Table 1:

\begin{tabular}{|c|c|c|c|c|c|c|c|}
\hline $\mathrm{CH}_{4}+$ & $\mathrm{Ar}$ & $\mathrm{Kr}$ & $\mathrm{Xe}$ & $\mathrm{Br}_{2}$ & $\mathrm{CCl}_{4}$ & $\mathrm{I}_{2}$ & $n-\mathrm{C}_{10} \mathrm{H}_{22}$ \\
\hline $10^{3} . \alpha_{T m \varepsilon \sigma}^{*}( \pm 5 \%)$ & -1.49 & -1.54 & -1.55 & -1.62 & -1.66 & -1.65 & -1.48 \\
\hline
\end{tabular}

